\title{
Genre et vulnérabilité au Sénégal : les femmes chefs de ménage
}

Mababou Kebe et Yves Charbit

\section{(2) OpenEdition}

1 Journals

Édition électronique

URL : https://journals.openedition.org/remi/4203

DOI : $10.4000 /$ remi.4203

ISSN : $1777-5418$

Éditeur

Université de Poitiers

Édition imprimée

Date de publication : 1 décembre 2007

Pagination : 51-65

ISBN : 978-2-911627-47-4

ISSN : 0765-0752

Référence électronique

Mababou Kebe et Yves Charbit, « Genre et vulnérabilité au Sénégal : les femmes chefs de ménage », Revue européenne des migrations internationales [En ligne], vol. 23 - n³ | 2007, mis en ligne le 01 décembre 2010, consulté le 14 avril 2022. URL : http://journals.openedition.org/remi/4203 ; DOI : https://doi.org/10.4000/remi.4203

Ce document a été généré automatiquement le 14 avril 2022.

() Université de Poitiers 


\title{
Genre et vulnérabilité au Sénégal : les femmes chefs de ménage
}

\author{
Mababou Kebe et Yves Charbit
}

\section{Ménages et pauvreté}

1 Au Sénégal, la mise en œuvre au début des années 1980 des politiques d'ajustement structurel a eu de lourdes conséquences sur les conditions de vie des populations. Non seulement leurs résultats économiques n'ont pas été à la hauteur des espérances, mais elles ont entraîné la paupérisation des populations et une grande vulnérabilité des ménages. Face à la crise, les familles ont fait preuve d'une grande capacité d'adaptation, qui s'est traduite par une diversification des modèles familiaux et une des évolutions majeures a été, au Sénégal comme ailleurs en Afrique subsaharienne, une proportion croissante des ménages dirigés par les femmes. Au Sénégal, on en dénombrait 19,4 \% en 2001. Quelle relation peut-on établir avec la pauvreté ?

2 Les analyses des rapports entre la pauvreté des ménages et leur féminisation se partagent entre deux positions extrêmes ${ }^{1}$. Pour certains chercheurs (Moghadam, 1996 ; Paolisso et Gammage, 1996), le fait, pour un ménage, d'avoir à sa tête une femme est source de pauvreté. Pour eux, la fragilité de la situation des femmes se répercute sur les ménages qu'elles dirigent et de ce fait ceux-ci sont plus sensibles à la pauvreté. Le second groupe, dans lequel on trouve Fonséca (1991) et Pilon (1996), postule au contraire que c'est la pauvreté qui détermine l'accès de la femme à la responsabilité d'un ménage. Il est difficile de trancher ce débat dans la mesure où l'établissement d'une causalité - et surtout de son sens - entre la pauvreté et le sexe du chef de ménage n'est pas aisé eu égard à l'hétérogénéité des ménages féminins, des conditions de leur formation et de leur fonctionnement (Garfinkel et McLanalah, 1986). Évoquons rapidement certaines des difficultés d'ordre méthodologique auxquelles se heurte la recherche sociodémographique et plus généralement quantitative.

3 Tout d'abord, que recouvre le concept de chef de ménage ? Le principal critère utilisé pour désigner le chef de ménage est le pouvoir économique. C'est le cas de Rosenhouse 
(1989) avec le concept de "working head", qui privilégie le membre du ménage le plus actif sur le marché du travail. Cependant, le pouvoir économique n'est pas toujours un critère décisif et il ne suffit pas d'être le membre ayant le plus de ressources pour être le chef du ménage et être reconnu comme tel par les autres membres. Par ailleurs, dans les grandes opérations de collecte démographique, les enquêteurs désignent souvent comme chef de ménage la personne qui leur fournit verbalement les informations sur le logement, la composition du ménage, etc. alors que celle qui s'auto-désigne chef de ménage le fait selon ses propres critères qui peuvent différer de ceux retenus par l'enquête. Enfin, les enquêtes sociodémographiques transversales, qui sont généralement utilisées pour étudier les relations entre le genre et la pauvreté, ne permettent pas de savoir si les conditions de vie des ménages, qui sont mesurées au moment même de l'enquête, sont simultanées, antérieures ou postérieures à l'arrivée de la femme à la tête du ménage. Or, les familles ne sont pas statiques et font l'objet de recompositions à l'occasion d'évènements démographiques majeurs (décès, migration, mariage...), d'ailleurs souvent liés à des facteurs économiques (exode rural à la recherche d'emploi par exemple).

4 Mais indépendamment de cette perspective "longitudinale ", l'étude des ménages féminins présente des difficultés spécifiques, car il y a incertitude sur l'attribution à une femme du statut de chef de ménage, les choix opérés étant souvent discutables. Dans la plupart des cas, en présence de son conjoint, la femme est systématiquement écartée de la responsabilité du ménage, alors qu'elle peut en être la vraie responsable (cas où le mari est inactif alors que la femme travaille). En principe la femme devient chef de ménage en cas d'absence physique du conjoint. Mais même alors, la femme peut être sous la responsabilité d'un autre homme du ménage (son père, un frère ou beaufrère, un fils, etc.), par exemple lorsque son conjoint est décédé. Et dans le cas où ce dernier est parti travailler à l'étranger, les enquêteurs attribuent parfois à la femme le statut de chef de ménage, alors que celui-ci est en fait dirigé par le migrant, qui en est en réalité le principal soutien économique et à qui reviennent les principales décisions concernant le ménage. En milieu urbain enfin, les ménages polygames sont contraints, faute d'espace, de se répartir entre plusieurs logements : ces pratiques de bi-résidence aboutissent à comptabiliser deux ménages, dont l'un est "attribué » à une des coépouses. En résumé, selon les objectifs de l'étude, la méthodologie, la perspicacité du chercheur, la proportion de femmes chef de ménage est sous-estimée ou au contraire surestimée.

Cet article est centré sur trois questions: les femmes chef de ménage ont-elles personnellement un profil sociodémographique source de vulnérabilité ? Quels sont les autres facteurs de vulnérabilité des ménages féminins? Et finalement ces ménages sont-ils plus ou moins vulnérables que ceux dirigés par des hommes?

Plusieurs grandes enquêtes, qui reposent sur des échantillons représentatifs au niveau national, permettent d'y répondre ${ }^{2}$. Nous utilisons les données de la deuxième phase de l'Enquête sénégalaise auprès des ménages de 2000-2001, qui a couvert 6594 ménages. La nature des données disponibles nous a conduit à privilégier la pauvreté monétaire des ménages. Nous avons pris pour indicateur la consommation finale des ménages, qui est un agrégat des dépenses des ménages au cours des douze derniers mois précédant l'enquête: dépenses alimentaires, consommation de biens non alimentaires et de certains services. Nous avons aussi défini une ligne ou un seuil de pauvreté à partir duquel les ménages vont être considérés comme pauvres ou riches, à partir de la 
méthode du coût des besoins de base a été utilisée pour estimer le seuil de pauvreté monétaire.

\footnotetext{
Calcul de l'indicateur de pauvreté monétaire

La méthode consiste d'abord à identifier un panier de biens de consommation courante à même de satisfaire les besoins nutritionnels quotidiens d'un individu, estimés entre 2100 et 2400 calories. Le choix du panier doit prendre en considération les habitudes alimentaires locales. Le seuil de pauvreté monétaire a été appréhendé sur une base par équivalent adulte pour réduire les biais pouvant être liés aux différences de taille et de composition des ménages. En l'absence de consensus sur cette question, la pondération retenue pour calculer le nombre d'équivalents adultes des ménages est celle qui est la plus utilisée : 0,5 pour les moins de 15 ans et 1 pour les personnes de plus de 15 ans (Ravallion, 1996).

La deuxième étape consiste à évaluer la valeur monétaire de ce panier en tenant compte des variations des prix entre les différentes régions et les différentes périodes considérées. Ce faisant, on obtient le seuil de pauvreté alimentaire auquel il faut ajouter le seuil de pauvreté non alimentaire pour obtenir le seuil de pauvreté global. En l'absence d'informations sur les coûts de la composante non alimentaire, on considère celle-ci comme une proportion du seuil alimentaire.

La consommation des ménages dont les dépenses se rapprochent le plus du seuil alimentaire est prise en compte pour estimer le seuil de pauvreté non alimentaire. Celui-ci consiste à la moyenne des dépenses non alimentaires par équivalent adulte des $5 \%$ de ces ménages qui se situent de part et d'autre de la ligne de pauvreté alimentaire. En raison de la faible taille de l'échantillon dans certaines régions, il n'a pas été possible d'ajuster les seuils de pauvreté en fonction de la région. Ils ont donc été calculés pour trois strates : Dakar, les villes secondaires, le monde rural. Notons pour information que si on compte au niveau national $19,4 \%$ des ménages féminins, on en trouve $24,7 \%$ dans la capitale, 30,4\% dans les autres villes du Sénégal, mais seulement $13 \%$ en milieu rural. Les seuils de pauvreté par équivalent adulte et par jour exprimés en FCFA ( 1 euro = 656 FCFA) sont les suivants. Dakar : 879 ; Autres villes : 713 ; Milieu rural : 498 .
}

\section{La vulnérabilité des femmes chefs de ménage}

Quelles sont les principales caractéristiques des femmes chef de ménage?

\section{La nuptialité}

8 On trouve les plus fortes proportions de femmes chefs de ménage parmi les personnes célibataires ou en rupture d'union; ainsi les chefs de ménage veufs ou divorcés sont majoritairement des femmes (respectivement $86,6 \%$ et $69,2 \%$ ) contre à peine $11,7 \%$ et $12,1 \%$ pour ceux qui vivent en union polygamique ou monogamique. Comme il s'agit ici de l'état matrimonial légal, il n'y a pas nécessairement cohabitation effective de l'homme et de son épouse, en particulier quand le mari parti en migration. Les femmes sont aussi surreprésentées parmi les jeunes chefs de ménages (moins de 25 ans). La précocité de la nuptialité et surtout de la fécondité des femmes (et plus particulièrement les naissances hors mariage) obligent les femmes à prendre en charge un ménage plus tôt que les hommes. Si l'on peut penser que l'âge et la nuptialité sont des facteurs de vulnérabilité, d'autres caractéristiques individuelles des femmes chef de ménage y contribuent : elles sont démunies en capital humain et faiblement intégrées au marché du travail ${ }^{3}$. 


\section{Une faible dotation en capital humain}

Comme leurs homologues masculins (et d'ailleurs l'ensemble de la population féminine sénégalaise), les femmes chef de ménage souffrent d'un faible niveau de scolarisation. En effet, quel que soit le sexe, les chefs de ménage se caractérisent par un faible niveau d'instruction. Cependant, l'analphabétisme est plus accentué chez les femmes (graphique 1). Le graphique 1 montre ainsi que seule une femme chef de ménage sur quatre est instruite. De même, 10,3\% seulement d'entre elles ont fait des études secondaires ou supérieures, contre $15,1 \%$ chez les hommes. L'absence ou la faiblesse de la scolarisation, combinée aux facteurs culturels qui confinent la femme dans son rôle de femme au foyer, entravent leur participation au marché du travail (graphique 1).

Graphique 1 : Répartition des chefs de ménage selon le sexe et le niveau d'instruction

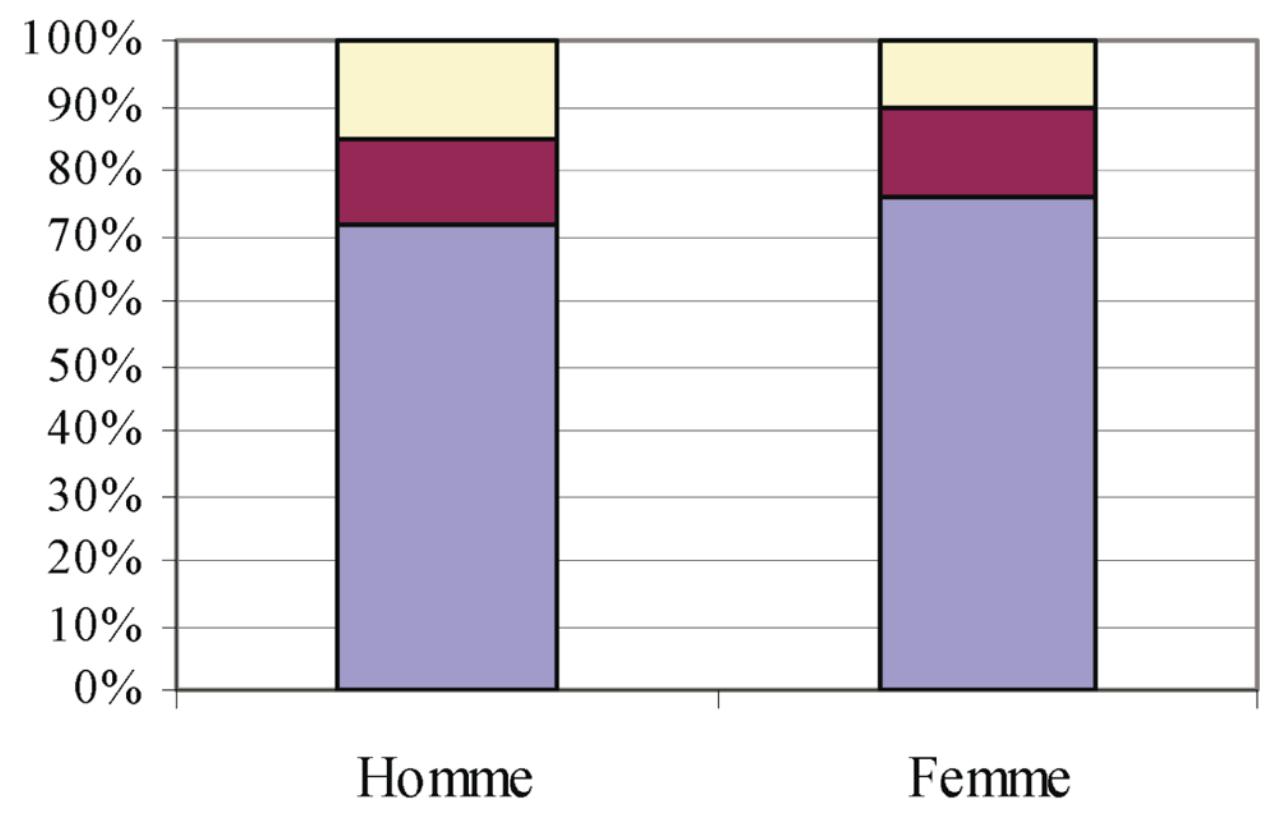

$\square$ Aucun $\square$ Primaire $\square$ Secondaire et plus 
Graphique 2 : Répartition des chefs de ménage selon le sexe et la participation au marché du travail

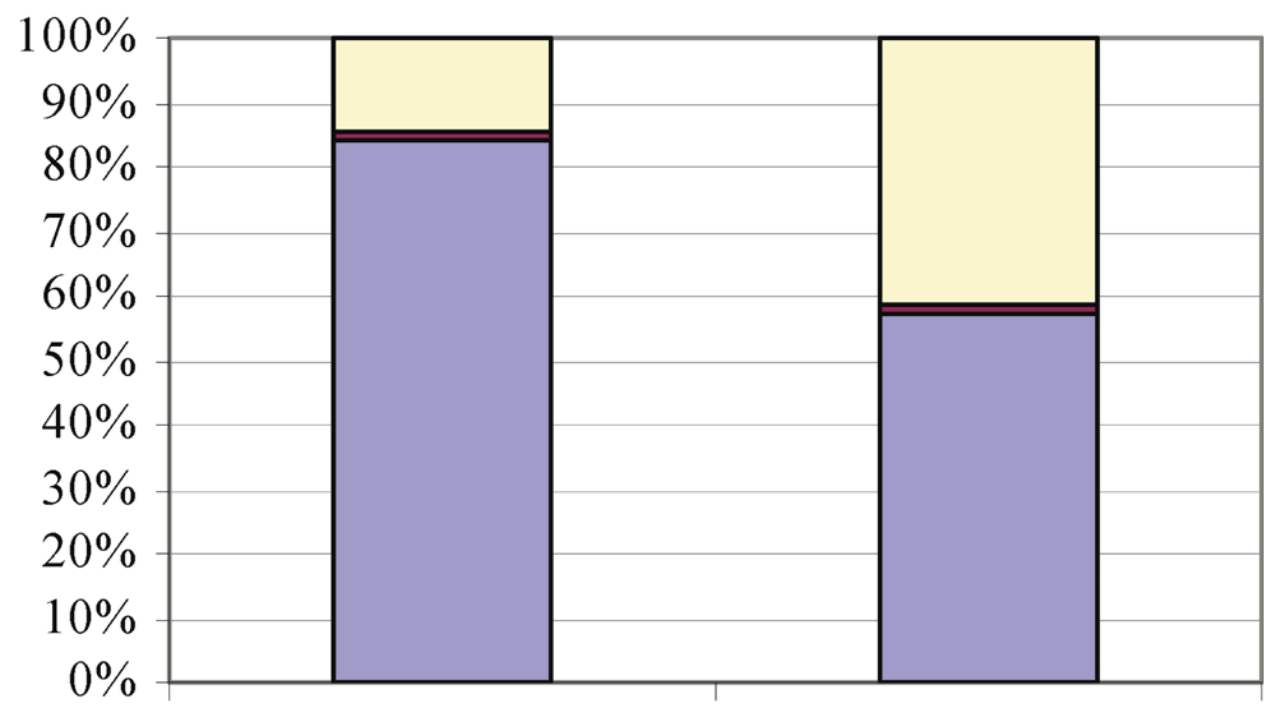

Homme Femme

\section{$\square$ Occupé $\square$ Chômeur $\square$ Inactif}

\section{Une faible intégration au marché du travail}

10 Les femmes chef de ménage sont faiblement intégrées au marché du travail. Non seulement une forte minorité en est exclue, mais celles qui y sont engagées sont cantonnées, pour la plupart, dans des activités faiblement productives (graphique 2).

11 Les femmes chef de ménage ont un taux d'activité sensiblement inférieur à celui des hommes : 59 \% contre 85,1\%. De même, parmi les actifs, 1,9\% des hommes sont en attente d'un emploi contre $2,8 \%$ des femmes; en outre, $31,2 \%$ des hommes chef de ménages occupés exercent un emploi du secteur secondaire contre $18,2 \%$ pour les femmes. Les femmes chef de ménage se caractérisent donc par une forte proportion de personnes non actives. Ces dernières, constituées en majorité de personnes au foyer $(61,6 \%)$ représentent $41 \%$ des femmes chef de ménage contre à peine $14,3 \%$ pour les hommes.

12 C'est dans la capitale que les taux d'inactivité et de chômage des femmes chef de ménage sont les plus importants. Alors que plus de $81 \%$ de leurs homologues masculins sont actifs, seule la moitié des chefs de ménage dakaroises sont occupées et parmi elles, $5 \%$ sont à la recherche d'un emploi. Un autre facteur de la faible intégration des femmes chef de ménage réside dans le fait qu'elles occupent les emplois les plus précaires et les moins rémunérateurs : $12 \%$ des femmes chef de ménages ont accès à une activité salariée contre $20,7 \%$ des hommes et leur revenu moyen est 4,8 fois moins élevé que celui des hommes.

13 Cette situation n'est pas sans incidences au niveau du fonctionnement des ménages, dans la mesure où le revenu du chef de ménage est un facteur décisif des conditions de vie de l'ensemble du ménage. Or, dans les ménages masculins, le revenu moyen du 
responsable est 14 fois plus élevé que celui des autres membres du ménage alors que l'écart est beaucoup plus faible (rapport de 3 à 1 seulement) dans les ménages féminins ${ }^{4}$. Cette différence réduit la marge de manœuvre des femmes chef de ménage par rapport à leurs homologues masculins, en leur conférant une moindre autorité dans les prises de décision relatives au fonctionnement du ménage.

La comparaison entre les hommes et les femmes chef de ménage montre donc que ces dernières sont plus défavorisées, que ce soit en termes d'instruction, d'emploi ou de revenus.

\section{La fragilité des ménages féminins}

15 Les caractéristiques individuelles des femmes chef de ménage sont aggravées par le fait que les femmes chef de ménage "héritent» des familles les plus fragiles. Outre l'absence d'un conjoint actif, les membres secondaires du ménage sont eux-mêmes, pour une grande part, des personnes vulnérables (jeunes, vieux, femmes...) et les revenus du ménage sont plus précaires que dans les ménages masculins.

\section{L'absence d'un conjoint actif}

Contrairement à leurs homologues masculins, elles ne peuvent presque jamais compter sur l'aide d'un conjoint: moins de $1 \%$ cohabite en effet avec leur mari. En outre, lorsque dans les ménages féminins le mari cohabite avec son épouse, ce dernier est dans la plupart des cas dans l'impossibilité de participer efficacement au fonctionnement du ménage, car il est très âgé : en moyenne 65,8 ans, alors que par exemple les hommes chef de ménage ont 50,2 ans. De même, parmi les hommes recensés comme vivant dans un ménage dirigé par leur épouse, seuls 40,8 \% d'entre eux sont occupés (contre $63,8 \%$ pour les épouses des hommes chef de ménage). La plupart d'entre eux sont retraités ou malades.

\section{Les caractéristiques des membres secondaires}

La population sénégalaise est très jeune : quel que soit le sexe du responsable, plus de la moitié des membres des ménages $(55 \%)$ a moins de 20 ans. Mais cette caractéristique classique des pays d'Afrique subsaharienne présente au Sénégal une forte asymétrie. Alors que dans les ménages masculins les membres, quel que soit leur sexe, ont pratiquement le même âge ( 21 ans en moyenne), dans les ménages féminins les hommes ont en moyenne 10 ans de moins que les femmes (10 ans contre 25 ans). Cette jeunesse de la population se reflète au niveau du rapport de dépendance est au désavantage des ménages féminins: on y relève 112 inactifs pour 100 actifs, contre 102 inactifs pour 100 actifs dans les ménages masculins.

La taille des ménages est une autre caractéristique défavorable aux femmes. Les ménages masculins regroupent en moyenne 2 membres de plus. Autre indicateur : les ménages de grande taille ( 15 personnes et plus), qui représentent plus de $17 \%$ des ménages, sont moins fréquents parmi ceux dirigés par les femmes (10,7\%). La taille plus réduite des ménages féminins peut constituer un facteur de vulnérabilité, car moins le ménage compte de membres, plus les conditions de vie vont être fortement dépendantes du revenu du chef de ménage. Dans les ménages masculins, qui ont une 
taille plus grande, d'autres membres peuvent contribuer ou suppléer le chef de ménage en cas de défaillance de celui-ci. Ils peuvent même être le principal soutien de la famille durant certaines périodes, telle la soudure entre deux récoltes.

\section{La précarité des revenus des ménages dirigés par les femmes}

19 Dans les ménages féminins, la participation économique des membres n'est pas très importante. Parmi la population en âge de travailler, $43,7 \%$ seulement sont actifs. De même, le taux de chômage y est très élevé, dans la mesure où $12 \%$ des personnes actives sont en attente d'emploi et de ce fait seul un petit nombre de personnes travaille et doit faire vivre toutes les autres. Les ménages dirigés par les hommes, où le taux d'activité de l'ensemble des membres avoisine $58 \%$, sont plus favorisés et leurs membres actifs sont moins touchés par le chômage $(4,7 \%)$. Mais surtout les revenus des ménages dirigés par des femmes sont de nature aléatoire et ponctuelle.

Les revenus des ménages proviennent de différentes sources, dont la plus importante sont les salaires des différents membres, qui représentent $30 \%$ du revenu, viennent ensuite les entreprises non agricoles, les transferts et la propriété. Dans les ménages masculins, les salaires des membres correspondent au tiers du revenu total, alors que dans les ménages féminins, la plus grande contribution est apportée par la propriété, mais comme les revenus de la propriété, contrairement au salaire, constituent une dépense en moins plutôt qu'une véritable rentrée de ressources, il peut en résulter des difficultés pour le ménage à acquérir des biens et des services. Les entreprises non agricoles constituent la deuxième source de revenu pour tous les ménages, qui assure pratiquement le quart des ressources des ménages. Une troisième source de revenus, aussi bien pour les ménages masculins que féminins, est constituée par les transferts, qui apportent $20 \%$ des revenus des ménages. La nature des sources de revenus, combinée à l'absence de soutien d'un conjoint, la composition des ménages et l'accès inégal aux ressources économiques rendent ainsi plus vulnérables les femmes chefs de ménages.

\section{La mobilisation des réseaux sociaux et la rente migratoire}

Cependant, pour pallier la faiblesse de leur revenu, les ménages dirigés par les femmes ont recours à la mobilisation des réseaux sociaux: la participation à des tontines et à d'autres formes de crédit, mais surtout les cadeaux et les dons provenant de personnes extérieures au ménage leur permettent de disposer de ressources d'appoint. Les cadeaux et dons échangés par les ménages constituent une pratique courante au Sénégal et les plus défavorisés peuvent souvent compter sur le soutien de ménages ou de personnes plus nantis. Cette solidarité s'exerce généralement au moment des fêtes et des cérémonies telles que les mariages, les baptêmes, les décès...

L'aide apportée est dans la grande majorité des cas, financière, mais les dons en nature sont loin d'être négligeables car ils représentent presque le tiers de la valeur des dons reçus, tandis que les repas ne représentent qu'une très faible partie des transferts volontaires vers les ménages. La nature des dons reçus par les ménages est la même quel que soit le sexe du responsable et à peu près autant de ménages en ont bénéficié (98\% contre 92,3\% dans les ménages masculins), mais ce sont les ménages féminins qui 
bénéficient le plus de cette solidarité, car le montant des cadeaux reçus est deux fois plus élevé.

Une source de revenus encore plus significative pour les ménages sénégalais réside dans les transferts liés à l'émigration interne ou internationale. Or, les ménages féminins se caractérisent par une plus grande proportion de migrants extérieurs : ils sont deux fois plus nombreux (18,4\% contre 9,6\%) à compter au moins un migrant international. Et surtout, l'aide apportée provient essentiellement de personnes qui vivent et travaillent dans les pays occidentaux, d'où sont envoyés les transferts monétaires les plus importantes et les plus réguliers. Si dans les ménages masculins c'est le lieu de résidence d'un peu plus de la moitié des expatriés $(51,9 \%)$, dans les ménages féminins cette proportion atteint $67,2 \%$ (tableau 1 ).

Tableau 1 : Destination des émigrés selon le sexe du chef de ménage

\begin{tabular}{|l|l|l|}
\hline & Ménages masculins & Ménages féminins \\
\hline Afrique & 45,6 & 30,2 \\
\hline Europe & 44,9 & 58,2 \\
\hline Amérique & 7,0 & 9,0 \\
\hline Autres & 2,6 & 2,6 \\
\hline Total & 100 & 100 \\
\hline
\end{tabular}

Encore faut-il que les migrants ne se désintéressent pas des ménages dont ils sont issus. Il apparait que ces derniers entretiennent des relations économiques étroites avec leurs familles : 73,9\% d'entre eux envoient de l'argent à leurs familles, dont 57,9\% le font de façon régulière et ces envois profitent un peu plus aux ménages féminins dont $76,2 \%$ des émigrés contribuent aux revenus des ménages, et parmi eux, $66,7 \%$ le font de façon régulière. Ces données confirment donc les théories de la dépendance réciproque entre le migrant et sa famille, qui expliquent le maintien des liens malgré la distance et le temps. 
Les interdépendances réciproques : individus et familles

La famille étant, dans les pays en développement, une instance bien plus influente sur les comportements des individus que dans les pays industrialisés, la décision de migrer comme celle de revenir au pays, doit s'analyser comme le résultat des interactions entre le migrant et sa famille, surtout s'il s'agit d'une famille étendue, à structure d'autorité verticale (Faist, 2000). D'où la conceptualisation des comportements en termes d'interdépendance réciproque.

Si l'on considère l'ensemble de l'unité économique constituée par la famille et le migrant, qui collaborent à court et à moyen terme, le gain économique est certain pour la famille, les transferts d'argent contribuant à la diversification des ressources du ménage. Une famille rurale qui veut passer de l'autosubsistance à une exploitation de type capitaliste est confrontée à l'absence d'accès au crédit, à l'impossibilité d'emprunter soit pour acheter de la terre, soit pour s'équiper en machines agricoles. C'est précisément ce rôle que jouent les transferts et que soulignent les enquêtes disponibles sur les utilisations des transferts, qui font mention, en milieu rural, de l'achat de terres. En d'autres termes l'unité familiale, à travers les gains escomptés de l'envoi d'un ou plusieurs actifs, procède bel et bien à une optimisation - ou au moins - à une ré-allocation de ses ressources.

Pour ce qui concerne l'intérêt du migrant individuel, il a bénéficié de la capacité de la famille à mobiliser un capital social : elle a rassemblé des ressources financières pour payer le départ et mobilisé ses réseaux familiaux ou communautaires pour prendre en charge le migrant (logement, démarches administratives, présentation et recommandation à des employeurs potentiels). Une enquête réalisée au Botswana de 1978-1979 (Stark, 1991 : 216-254) montre que les échanges et retombées sont échelonnés dans le temps et que les transferts augmentent en fonction de trois variables : le niveau d'instruction, la taille des troupeaux, l'intensité des périodes de sécheresse. Les transferts sont un investissement dans l'éducation des jeunes pour assurer des ressources ultérieures (mais ici la relation peut être inverse). Les troupeaux étant la principale forme d'héritage, le migrant a tout intérêt à marquer sa prétention à l'héritage. Enfin, l'envoi d'argent en raison de la sécheresse ne s'analyse pas comme pas un acte d'altruisme pur, mais un moyen de limiter le risque d'hériter de bêtes mortes de faim et de soif.

Pour que le système fonctionne, il faut donc que les liens entre le migrant et la famille soient suffisamment forts. L'hypothèse d'une rupture unilatérale du contrat est-elle envisageable? Le risque est loin d'être nul, si le migrant met en œuvre une autre stratégie (mariage mixte et intégration définitive à l'étranger), mais la force des liens culturels et identitaires - impossibles à quantifier économiquement - va se traduire par une logique de retour ou de va-et-vient. Ainsi, le non-respect du contrat familial peut exclure le migrant du marché matrimonial local, voire de la communauté. Le risque de non-retour est bien sûr plus élevé pour un migrant célibataire que pour un homme marié.

(Source : d'après Charbit, à paraître 2008).

Le bilan penche-t-il finalement en faveur des femmes? Pour répondre à cette question classons les ménages selon leur type, le sexe du chef de ménage, et la prévalence de la pauvreté.

26 Si plus de la moitié des ménages sénégalais $(53,1 \%)$ vivait en dessous du seuil de pauvreté en 2000-2001, la prévalence de la pauvreté était moins importante dans les ménages féminins où elle concernait $42,6 \%$ des ménages (contre $55,7 \%$ pour les ménages masculins). Si l'on s'intéresse à la distribution de la pauvreté selon le type de ménage, on remarque que les moins affectés par la pauvreté sont les deux catégories de "ménage monoparental » $(30,5 \%$ de pauvres) et surtout de "chef de ménage isolé » ( $7 \%$ de pauvres), contre $43,4 \%$ pour les ménages nucléaires et $57 \%$ pour les ménages étendus. Cependant, dans les deux premières catégories, qui dépendent plus 
étroitement des caractéristiques individuelles de leur responsable, ce sont les ménages féminins qui sont plus affectés par la pauvreté. Dans les ménages nucléaires ou étendus, la vulnérabilité des femmes chef de ménage est sans doute contrebalancée par l'apport des autres membres du ménage et plus précisément la présence de membres secondaires actifs a une incidence positive sur les conditions de vie des ménages féminins.

Graphique 3 : Incidence de la pauvreté selon le sexe du chef de ménage et le type de ménage

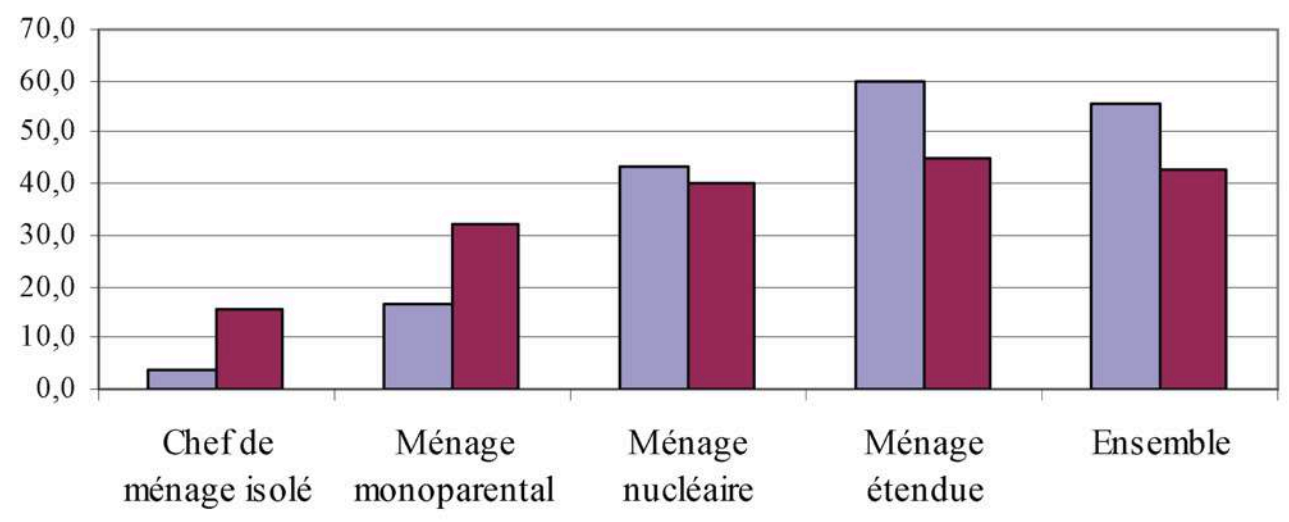

$\square$ Homme $\square$ Femme

\section{Féminisation de la pauvreté ?}

Les femmes chef de ménage souffrent de handicaps plus sérieux que les hommes : elles sont plus souvent urbaines, analphabètes, et jeunes ce sont aussi des femmes âgées et veuves. Lorsqu'elles sont engagées dans le secteur économique, elles sont dans la plupart des cas cantonnées dans des activités précaires et faiblement productives. La vulnérabilité des femmes chef de ménage est aussi liée aux caractéristiques de leur ménage et au profil des membres secondaires : pas de conjoint qui puisse contribuer à l'économie du ménage, des dépendants et des inactifs plus nombreux. Cet article fondé sur des sources quantitatives ne pouvait envisager la dimension socioculturelle de cette réalité, mais il est probable que ces femmes continuent d'être confinées dans une position subalterne et que leur rôle de chef de ménage a peu d'incidence sur leur statut. Dans la plupart des cas, le rôle de chef de ménage confère à la femme de lourdes responsabilités et charges y afférant, mais non le statut et les prérogatives qui y sont liés.

La question est de savoir si tous ces facteurs défavorables se traduisent par une pauvreté plus aiguë. Le recours à un indicateur de pauvreté monétaire aboutit à un résultat majeur : les ménages féminins sont moins exposés à la pauvreté monétaire que ceux dirigés par les hommes et c'est la mobilisation des réseaux sociaux (aide apportée par d'autres ménages et transferts des migrants) qui permet aux ménages féminins de compenser tous les autres facteurs de vulnérabilité. Les relations économiques qu'elles entretiennent avec des personnes extérieures aux ménages, et plus particulièrement les migrants internationaux, sont ici décisives pour l'amélioration de leurs conditions de vie. 
Si leur vulnérabilité est donc moindre que celles des chefs de ménage masculins, on ne saurait, pour autant, en déduire un processus « d'empowerment ». Ce terme difficilement traduisible en français, exprime un processus global d'amélioration du statut de la femme, entendu dans le sens d'une plus grande autonomie par rapport à son conjoint, sa famille d'origine, sa communauté d'appartenance, la société globale, et ceci au niveau psychologique, social, culturel, économique ou enfin politique.

L'enjeu est celui de la dépendance. Les dons reçus par les ménages sont une source de revenus qui permet certes de réduire temporairement leurs difficultés, mais ils ne peuvent garantir un fonctionnement régulier du ménage. Et dans la mesure où les femmes sont obligées de recourir à des réseaux de solidarité pour pouvoir satisfaire les besoins du ménage, elles risquent de se trouver enfermée dans une relation d'assistance. S'il est vrai que celle-ci est culturellement et socialement acceptée, elle ne contribue pas à l'autonomie des femmes chef de ménage. La rente migratoire pose, elle, un autre problème quant à une éventuelle évolution du statut des femmes liée à une diminution de la pauvreté. Si la gestion des transferts d'argent par les maris qui travaillent à l'étranger est contrôlée par d'autres qu'elles, elles ne bénéficieront pas d'une marge de manœuvre accrue dans la prise des décisions relatives au fonctionnement des ménages. Tout dépend donc finalement des conditions dans lesquelles l'économie des ménages s'inscrit dans le culturel et le social.

\section{BIBLIOGRAPHIE}

ANTOINE Philippe, NANITELAMIO Jeanne (1990) Nouveaux statuts féminins et urbanisation en Afrique, Genus, Vol XLVI n 3-4, pp. 17-30.

BADEN Sally, MILWARD Kirsty (1995) Gender and poverty, Bridge Report, $\mathrm{n}^{\circ}$ 30, Brighton : Institute of Development Studies.

BISILLIAT Jeanne (dir.) (1996) Femmes du Sud, chefs de famille, Paris, Karthala.

BUVINIC Mayra, GUPTA Geeta Rao (1997) Female-headed households and Female-maintained families : are they worth targeting to reduce poverty in developing countries ?, Economic Development and Cultural Change, vol. 45, $\mathrm{n}^{\circ}$ 2, January, pp. 259-280.

BUVINIC Mayra, GUPTA Geeta Rao (1994) Targeting poor woman-headed households and womanmaintained families in developing countries : views on a policy dilemma, ICRW/ Population Council program on female headship and poverty in developing countries.

CAIRE Guy (1989) Analyse microéconomique du travail féminin : apports et apories, Revue d'Economie Politique, 99e année, $n^{\circ} 3$, pp. 446-465.

CHANT Sylvia (1997) Women-headed households : poorest of the poor ? Perspectives from Mexico, Costa Rica and Philippines, Institute of Development Studies (IDS), vol. 28, $\mathrm{n}^{\circ} 3$, July, pp. 26-48. 
CHARBIT Yves (2008) Transferts, retours et développement : données, concepts et problématiques, in Véronique Petit Éd. : Les migrations internationales de retour dans la perspective des pays du sud, pp. 44-75.

CHIAPPORI Pierre-André (1992) Collective models of household behaviour : the sharing rule approach, International Food Policy Research Institute, World Bank, vol. 14, pp. 245-255.

COQUERY-VIDROVITCH Catherine (1994) Les Africaines, histoire de la femme en Afrique Noire du XIX au $\mathrm{XX}^{\mathrm{e}}$ siècle, Paris, Desjonquières.

FAIST Thomas (2000) The crucial meso-level, in The Volume and Dynamics of International Migration and Transnational Social Spaces, Oxford University Press, Oxford, pp. 187-217.

FONSECA Claudia (1991) Spouses, siblings and sex-linked bonding : a look at kinship organisation in a Brazilian slum, JELIN E., ed., Family, Household and gender relations in Latin America, London : Kegan Paul International/Paris : Unesco, pp. 133-160.

GARFINKEL Irwin, McLANAHAN Sara (1986) Single mothers and their children : a new American dilemma, Washington, Urban Institute Press.

HAINARD François, VERSCHUUR Christine (2001) Femmes dans les crises urbaines, Paris, Karthala.

KIMENYI Mwangi S., MBAKU John Mkum (1995) Female headship, feminization of poverty and welfare, Southern Economic Journal, 62, 1, July, pp. 44-52.

KUMARI Ranjana (1989) Women-headed households in rural India, New Delhi : Radiant Publishers.

LACHAUD Jean-Pierre (1998) Pauvreté et Mauritanie : une approche multidimensionnelle, Centre d'économie du développement (CED), Université Montesquieu - Bordeaux IV.

LLOYD Cynthia B., BRANDON Anastasia J. (1993) Women's role in maintaining households : family welfare and sexual inequality in Ghana, Population studies, vol. 47, pp. 115-131.

MARCOUX Alain (1997) The feminization of poverty : facts, hypotheses and the art of advocacy, FAO, Women and population division.

MOGHADAM Valentine (1996) The feminization of poverty : notes on a concept and trends, report prepared for the United Nations Development Programme, Human Development Report, September.

PAOLISSO Michael, GAMMAGE Sarah (1996) Women's responses to environmental degradation : case studies from Latin America, Washington DC : International Centre for Research on women.

PEARCE Diana M. (1978) The feminisation of poverty : women, work and welfare, Urban and Social Change Review, February, pp. 28-36.

PILON Marc, MAMA Mouhamadou, TICHIT Christine (1997) Les femmes chefs de ménage en Afrique : aperçu général et études de cas, in : PILON Marc, LOCOH Thérèse, VIGNIKIN Koukou E. et VIMARD Patrice (dir.), Ménages et familles en Afrique: Approches des dynamiques contemporaines, Les études du CEPED, $\mathrm{n}^{\circ}$ 15, Paris, CEPED, pp. 167-191.

PILON Marc (1996) Les femmes chefs de ménage en Afrique : état des connaissances, In BISILLIAT, Jeanne dir., Femmes du Sud, chefs de familles, Khartala, pp. 236-256.

RAVALLION Martin (1996) Comparaisons de la pauvreté : concepts et méthodes. Banque mondiale, Document de travail LSMS.

ROSENHOUSE Sandra (1989) Identifying the poor : is headship a useful concept ?, Living Standard Measurement Study, n 58, 50 p., World Bank. 
STARK Oded (1991) The Migration of labour, Basil Blackwell, $406 \mathrm{p}$.

TICHIT Christine (2002) Les femmes chefs de ménage au Cameroun : entre autonomie, monoparentalité et isolement, Thèse de doctorat, Université Paris X-Nanterre, $441 \mathrm{p}$.

\section{NOTES}

1. On trouvera en bibliographie quelques références utiles sur le sujet. Par commodité, on désignera respectivement par ménages féminins ceux dont le chef est une femme et par ménages masculins ceux qui ont à leur tête un homme. Les uns et les autres comportent bien évidemment des membres des deux sexes.

2. Le système d'information sénégalais sur les conditions de vie des ménages est très riche par rapport à d'autres pays d'Afrique subsaharienne. Les chercheurs disposent de l'Enquête sénégalaise auprès des ménages (ESAM 1994-1995 et ESAM 2000-2001), l'Enquête sur les priorités (ESP, 1991-1992) et l'Enquête sur les dépenses des ménages de la capitale (EDMC, 1996). Mais si l'existence de telles bases de données constitue un apport inestimable, elles demeurent encore largement sousutilisées.

3. Lachaud (1998) note ainsi que la participation au marché du travail et le niveau des dotations en capital humain constituent les deux éléments les plus efficaces pour réduire la pauvreté.

4. Les données sur le revenu proviennent de la première phase de l'enquête sénégalaise auprès des ménages de 1994-1995, la deuxième enquête n'ayant pas collecté des informations de cette nature.

\section{RÉSUMÉS}

La pauvreté monétaire de 6594 ménages est analysée à partir de l'Enquête sénégalaise auprès des ménages de 2000-2001.

Les femmes chef de ménage souffrent de handicaps plus sérieux que les hommes : elles sont plus souvent urbaines, analphabètes, plus jeunes. Ce sont aussi des femmes âgées et veuves. Leur plus faible dotation en capital humain et une médiocre intégration au marché du travail se traduisent par des activités précaires et faiblement productives. En outre, la vulnérabilité des ménages dirigés par des femmes est attribuable à l'absence d'un conjoint actif, au fait que les membres secondaires du ménage sont eux-mêmes, pour une grande part, des personnes vulnérables (jeunes, vieux, femmes...) et enfin à des revenus plus précaires que dans les ménages masculins. Cependant la mobilisation des réseaux sociaux et surtout la rente migratoire compensent ces handicaps, car les ménages féminins se caractérisent par une plus grande proportion de migrants internationaux, qui de plus travaillent dans les pays occidentaux, d'où sont envoyés les transferts monétaires les plus importantes et les plus réguliers.

Gender and Vulnerability among Senegalese Women Heads of Households. The monetary poverty of 6594 households is analysed using data collected during the 2000-2001 Enquête sénégalaise auprès des ménages. Women heads of households suffer from more serious handicaps than men, being more often urban illiterate and young and when they are widowed, they are also older. As they benefit from a smaller amount of human capital and are poorly integrated into the 
labour market, their economic activities are precarious and poorly productive. Moreover, the higher vulnerability of their households is due to the lack an active spouse, to a larger proportion of vulnerable persons (more young and old-aged persons, more women), and last to more precarious incomes. However their capacity to mobilize their social networks and above all their migratory rent compensate these handicaps, since female-headed households send more migrants abroad, especially to Western countries from where remittances are larger and more stable.

Genero y vulnerabilidad en Senegal: mujeres cabeza de familia. La pobreza monetaria de 6.594 hogares es analizada a partir de la Encuesta senegalesa de hogares de 2000-2001.

Las mujeres cabeza de familia sufren handicaps más serios que los hombres: son, más a menudo, urbanas, analfabetas y más jóvenes. Se trata también de mujeres ancianas y viudas. Su débil dotación en capital humano y su integración mediocre en el mercado laboral se traducen en actividades precarias y poco productivas. La vulnerabilidad de los hogares dirigidos por mujeres se atribuye a la ausencia de un compañero activo, al hecho de que los miembros secundarios del hogar son en su mayoría personas vulnerables (jóvenes, ancianos, mujeres,...) y, por último, a ingresos más precarios que en los hogares con presencia masculina.

Sin embargo, la movilización de las redes sociales y sobre todo la renta migratoria compensan estos handicaps. Los hogares femeninos se caracterizan por una mayor proporción de emigrantes internacionales que, además, trabajan en países occidentales desde donde son enviadas las transferencias monetarias más importantes y regulares.

\section{AUTEURS}

\section{MABABOU KEBE}

Agent de recherche, Institut national de santé publique du Québec. mababou.kebe@inspq.qc.ca 